\title{
Z-contrast HAADF-STEM Tomography
}

\author{
P.A.Midgley, M. Weyland, L. Laffont and J.M. Thomas
}

Department of Materials Science and Metallurgy, University of Cambridge, Pembroke Street, Cambridge, CB2 3QZ, UK

Whilst electron tomography has been used widely in the life sciences for decades it is only in the last few years that electron tomography has been considered in the physical sciences. The requirement for three-dimensional imaging has been driven by the need to investigate nano-structured devices and materials in which the lateral dimensions of features of interest are comparable to their depth. BF tomography is, in general, unsuitable for examination of specimens in the physical sciences. The prevalence of diffraction contrast in many crystalline specimens negates the use of BF images as they no longer satisfy the 'projection criterion', used as the basis for tomographic reconstruction. Instead, tomographers in the physical sciences have turned to imaging techniques in which the signal can be regarded as arising from incoherent scattering processes; images formed this way will not show diffraction contrast. One technique is energy-filtered TEM (EFTEM) and this will be discussed elsewhere in these Proceedings. Another is STEM high angle annular dark field (HAADF) imaging shown schematically in Fig. 1 [1]. The electrons are collected at high scattering angles (typically $>30$ mrad) using an annular detector. This technique excludes almost all of the low angle scattered coherent electrons making such images 'true projections' of structure, one of the pre-requisites for tomography. To a good approximation, the intensity of the images formed using high angle scattered electrons is modelled by Rutherford scattering: the intensity is proportional to the square of the atomic number, $Z^{2}$. In addition, the scanning nature of the STEM process enables beam-sensitive samples to be examined for far greater time periods than using conventional fixed-beam TEM [2].

In order to reach high tilt angles in a high resolution analytical TEM new holders have had to be designed which allow high tilt $\left(+/-80^{\circ}\right)$ within a narrow pole-piece gap. For all tomography experiments we have used either an FEI CM300 FEG or Tecnai F20 both having a Super-Twin lens with a pole-piece gap of $5.4 \mathrm{~mm}$. In the latest experiments we have used a Fischione-designed ultrahigh tilt holder. Tilt series are acquired using only a single axis with images acquired every $2^{\circ}$ and with a tilt range of $+/-60^{\circ}$ or $+/-70^{\circ}$ to minimise elongation in the reconstruction.

As an example of STEM tomography, Figs 2 and 3 show results from a reconstruction of a magnetotactic bacterium showing both the outer wall of the bacterial membrane and the 'backbone' of magnetite nanocrystals, used by the bacterium for sensing changes in the earth's gravitational magnetic field. The reconstruction was undertaken using an iterative back-projection routine that constrains the final reconstruction to match the original projection [3]. This has the effect of minimising artefacts introduced through the back-projection algorithm. The faceting of the nanocrystals is revealed very clearly in sections taken through the crystal marked $X$ in Fig. 2 . Fig. 3 (a) shows the truncated nature of the nanocrystal near its end emphasising the $\{111\}$ facets. 3(b) taken from the centre of the nanocrystal reveals the prismatic habit of the crystal, showing the equality of the six $\{110\}$ facets. There is very little artefactual intensity in the slice indicating the fidelity of the reconstruction.

STEM HAADF tomography promises to be an exciting new tool for the three-dimensional analysis of many nano-scale structures in the physical sciences [4]. 
References

[1] P.A. Midgley and M. Weyland, Ultramicroscopy (2003), in press.

[2] P.A. Midgley et al., Chem. Comm. 18 (2001) 907.

[3] P.F.C. Gilbert, Proc. R. Soc. Lond. B. 182 (1972) 89.

[4] This research was funded by the EPSRC and the Royal Commission for the Exhibition of 1851.
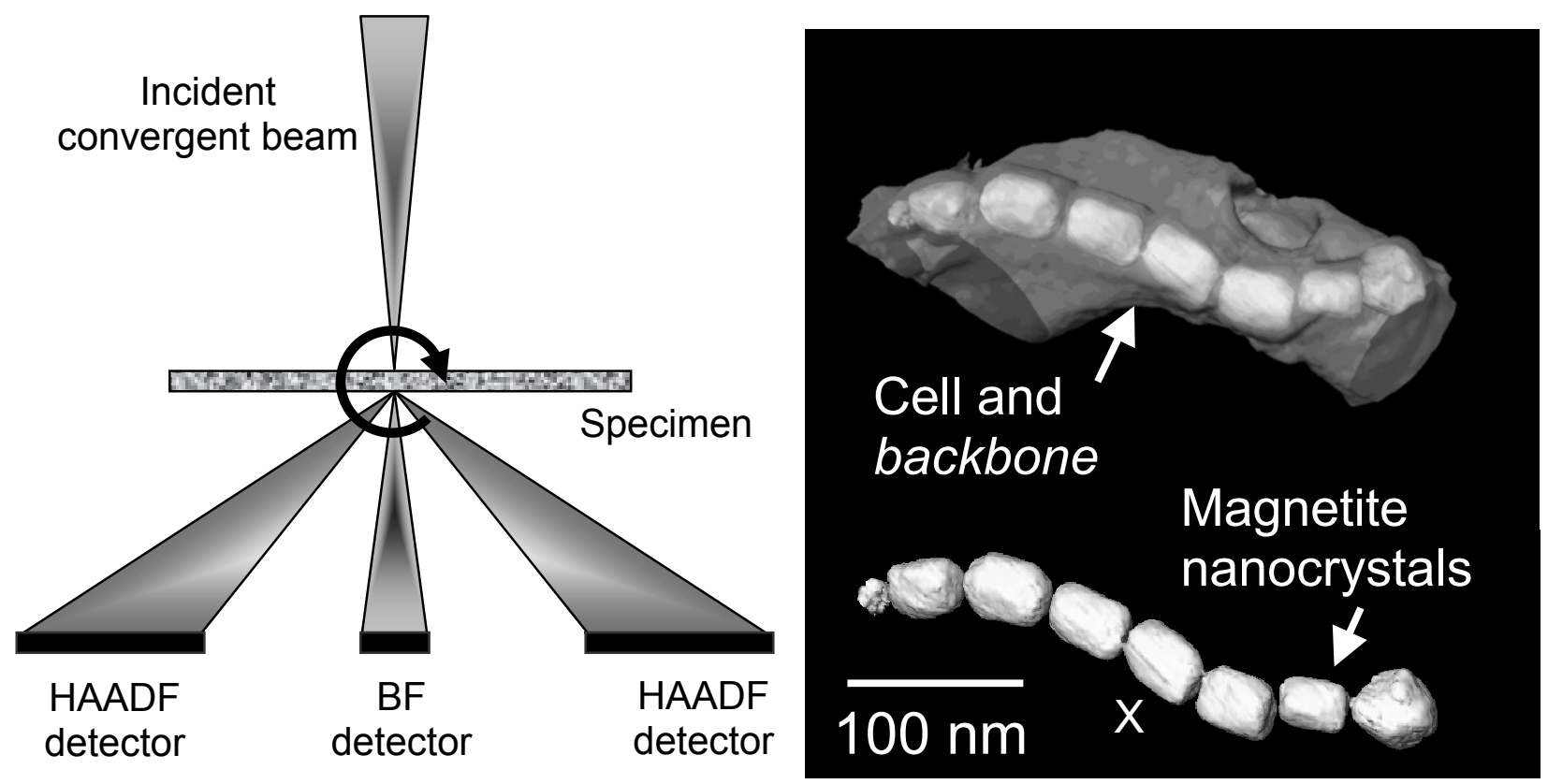

FIG 1. Schematic of a typical STEM detector arrangement. The angular range of the $\mathrm{BF}$ detectors is typically $0-5 \mathrm{mrad}$, for the HAADF detector this is $30-150 \mathrm{mrad}$.

FIG 2. Tomographic reconstruction of a magnetotactic bacterium showing the outline of the cell remnants together with the magnetite nanocrystal 'backbone'.
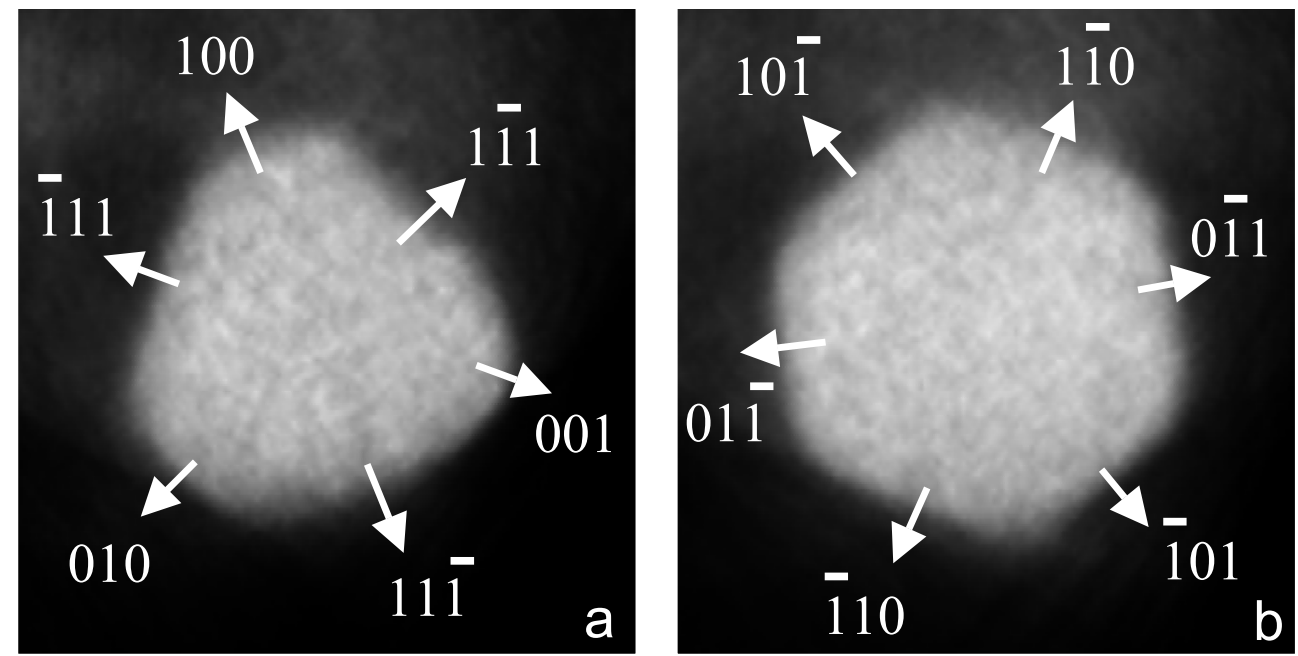

FIG 3. Sections through the magnetite crystal indicated in Fig. 2. (a) shows the truncated nature of the nanocrystal near its end emphasising the $\{111\}$ and $\{100\}$ facets. (b) taken from the centre of the nanocrystal reveals the prismatic habit of the crystal, showing the equality of the six $\{110\}$ facets. 